\title{
Bedtime ingestion of hypertension medications reduces the risk of new-onset type 2 diabetes: a randomised controlled trial
}

\author{
Ramón C. Hermida $^{1}$ • Diana E. Ayala ${ }^{1}$ • Artemio Mojón ${ }^{1}$ - José R. Fernández ${ }^{1}$
}

Received: 27 April 2015 / Accepted: 19 August 2015 / Published online: 23 September 2015

(C) Springer-Verlag Berlin Heidelberg 2015

\begin{abstract}
Aims/hypothesis We investigated whether therapy with the entire daily dose of $\geq 1$ hypertension medications at bedtime exerts greater reduction in the risk of new-onset diabetes than therapy with all medications upon awakening.

Methods We conducted a prospective, randomised, open-label, blinded endpoint trial of 2,012 hypertensive patients without diabetes, 976 men and 1,036 women, $52.7 \pm 13.6$ years of age. Patients were randomised, using a computer-generated allocation table, to ingest all their prescribed hypertension medications upon awakening or the entire daily dose of $\geq 1$ of them at bedtime. Investigators blinded to the hypertension treatment scheme of the patients assessed the development of new-onset diabetes.

Results During a 5.9-year median follow-up, 171 participants developed type 2 diabetes. Patients of the bedtime, compared with the morning-treatment group, showed: (1) significantly lower asleep BP mean, greater sleep-time relative BP decline and attenuated prevalence of non-dipping at the final evaluation ( $32 \%$ vs $52 \%, p<0.001)$; and (2) significantly lower HR of new-onset diabetes after adjustment for the significant influential characteristics of fasting glucose, waist circumference, asleep systolic BP mean, dipping classification and chronic kidney disease (CKD) (unadjusted HR 0.41 [95\% CI 0.29, 0.58]; adjusted HR 0.43 [0.31, 0.61]; event-rate $4.8 \%$ vs $12.1 \%$ with bedtime and morning treatment, respectively; $p<0.001$ ). Greater benefit was observed for bedtime
\end{abstract}

Ramón C. Hermida

rhermida@uvigo.es

1 Bioengineering and Chronobiology Laboratories, E.I.

Telecomunicación, University of Vigo, Campus Universitario, Vigo, Pontevedra 36310, Spain compared with awakening treatment with angiotensin receptor blockers (ARBs) (HR 0.39 [0.22, 0.69]; $p<0.001$ ), ACE inhibitors $(0.31[0.12,0.79], p=0.015)$ and $\beta$-blockers $(0.35$ $[0.14,0.85], p=0.021)$.

Conclusions/interpretation In hypertensive patients without diabetes, ingestion of $\geq 1$ BP-lowering medications at bedtime, mainly those modulating or blocking the effects of angiotensin II, compared with ingestion of all such medications upon awakening, results in improved ambulatory BP (ABP) control (significant further decrease of asleep BP) and reduced risk of new-onset diabetes.

Trial registration: ClinicalTrials.gov NCT00295542

Funding: This independent investigator-promoted research was supported by unrestricted grants from Ministerio de Ciencia e Innovación (SAF2006-6254-FEDER; SAF20097028-FEDER); Xunta de Galicia (PGIDIT03-PXIB32201PR; INCITE07-PXI-322003ES; INCITE08-E1R322063ES; INCITE09-E2R-322099ES; 09CSA018322PR); and Vicerrectorado de Investigación, University of Vigo.

Keywords Ambulatory blood pressure monitoring · Bedtime therapy $\cdot$ Renin-angiotensin blockade $\cdot$ Sleep-time blood pressure · Type 2 diabetes

$\begin{array}{ll}\text { Abbreviations } \\ \text { ABP } & \text { Ambulatory BP } \\ \text { ABPM } & \text { Ambulatory BP monitoring } \\ \text { ACEI } & \text { ACE inhibitor } \\ \text { ARB } & \text { Angiotensin receptor blocker } \\ \text { CCB } & \text { Calcium channel blocker } \\ \text { CKD } & \text { Chronic kidney disease } \\ \text { CKD-EPI } & \text { CKD Epidemiology Collaboration } \\ \text { CVD } & \text { Cardiovascular disease } \\ \text { DBP } & \text { Diastolic BP }\end{array}$




$\begin{array}{ll}\text { DREAM } & \begin{array}{l}\text { Diabetes Reduction Assessment with } \\ \text { Ramipril and Rosiglitazone Medication }\end{array} \\ \text { HOPE } & \begin{array}{l}\text { Heart Outcomes Prevention Evaluation } \\ \text { Monitorización Ambulatoria para Predicción } \\ \text { de Eventos Cardiovasculares }\end{array} \\ \text { PP } & \begin{array}{l}\text { Pulse pressure } \\ \text { Prospective, randomised, open-label, blinded } \\ \text { PROBE }\end{array} \\ \text { endpoint }\end{array}$

\section{Introduction}

Hypertension commonly occurs in conjunction with insulin resistance. Activation of the renin-angiotensin-aldosterone system (RAAS) and consequent elevations of angiotensin II and aldosterone contributes to increased hepatic glucose release and decreased insulin sensitivity [1]. Moreover, hyperglycaemia also increases serum aldosterone levels. Accordingly, in addition to BP-lowering, RAAS blockade might also serve as an effective strategy to control impaired glucose and insulin tolerance. Indeed, several large-scale clinical trials reviewed elsewhere $[2,3]$ have suggested ingestion of angiotensin receptor blockers (ARBs) or ACE inhibitors (ACEIs) might reduce the incidence of new-onset diabetes in hypertensive patients. In the Heart Outcomes Prevention Evaluation (HOPE) trial, add-on bedtime ACEI ramipril therapy, relative to placebo, not only significantly reduced the primary outcome variables of cardiovascular disease (CVD) death, myocardial infarction and stroke [4], but also the risk of new-onset diabetes by $34 \%$ [5]. However, the Diabetes Reduction Assessment with Ramipril and Rosiglitazone Medication (DREAM) trial, in which participants were also randomised to ramipril vs placebo but without a mandatory treatment-time indication, did not corroborate the benefit of ramipril for newonset diabetes prevention [6].

Regarding time-of-day of hypertension treatment, multiple clinical trials extensively reviewed elsewhere $[7,8]$ have detected prominent morning-evening, treatment-time differences in BP-lowering efficacy, duration of action, safety profile and/or effects upon the $24 \mathrm{~h}$ ambulatory BP (ABP) pattern of different classes of hypertension medications and their combinations. In particular, since the RAAS is highly circadian rhythmic and activates during night-time sleep [9], the once-daily bedtime, in comparison with upon-awakening, ingestion schedule of ARBs and ACEIs results in greater therapeutic effect on asleep BP mean determined by ABP monitoring $(\mathrm{ABPM})$, independent of terminal half-life $[7,8,10]$. This is the case, also, for the bedtime vs morning regimen of the ARB-calcium channel blocker (CCB), ACEI-CCB and ARBdiuretic combinations so far tested $[7,8]$.
The impact of hypertension treatment-time on asleep BP regulation might be clinically relevant based on the findings of numerous independent prospective studies that demonstrate elevated sleep-time BP constitutes a significant CVD risk factor that is independent of daytime clinic BP and the ABPMderived awake or $24 \mathrm{~h}$ BP means, both in patients without [11-16] and with diabetes [17-19]. Most important, sleeptime ABP, but not awake ABP or clinic BP, has also been identified as a highly significant prognostic marker of newonset diabetes [20]. Along these lines, the MAPEC study (Monitorización Ambulatoria para Predicción de Eventos Cardiovasculares, i.e., Ambulatory Blood Pressure Monitoring for Prediction of Cardiovascular Events), the first prospective randomised treatment-time investigation testing the worthiness of bedtime chronotherapy with $\geq 1$ conventional hypertension medications to specifically target attenuation of asleep ABP, demonstrated, relative to conventional morning therapy, significantly better reduction of CVD risk [21], also in high-risk patients with diabetes [22], chronic kidney disease (CKD) [23], or resistant hypertension [24]. The HR of CVD events was higher in patients randomised to treatment upon awakening, no matter the classes of BP-lowering medications prescribed. Moreover, among patients randomised to take $\geq 1$ medications at bedtime, ARB therapy was associated with significantly lower CVD risk than any other class of therapy [25]. However, whether timed hypertension treatment also influences the risk of new-onset diabetes is unknown.

These collective findings lead us to propose two hypotheses that have never before been prospectively investigated: (1) bedtime therapy with the entire daily dose of $\geq 1$ hypertension medications offers better protection against development of new-onset diabetes than morning-time therapy with all medications; and (2) RAAS inhibition or blockade is superior to any other treatment strategy for achieving such. The MAPEC study, specifically designed to prospectively investigate whether bedtime compared with upon-awakening BP-lowering treatment results in reduced risk of CVD, new-onset diabetes and renal events, allowed us to test these hypotheses.

\section{Methods}

Inclusion and exclusion criteria Complete details of the rationale and design of the MAPEC study are described in previous publications [14, 15, 19, 21-24]. In summary, the sample of the MAPEC study consisted of Spanish individuals $\geq 18$ years of age who adhered to a routine of daytime activity and night-time sleep. Inclusion criteria required participants to be either normotensive, untreated hypertensive or resistant to treatment [26] when ingesting all their prescribed BPlowering medications upon awakening. Exclusion criteria were pregnancy, drug/alcohol abuse history, night/shift-work employment, AIDS, type 1 diabetes, secondary hypertension, 
CVD disorders (unstable angina pectoris, heart failure, lifethreatening arrhythmia, nephropathy and grade III-IV retinopathy), intolerance to ABPM, and inability to communicate and comply with all study requirements. This prospective singlecentre study (ClinicalTrial.gov registration no. NCT00295542) was approved by the state Ethics Committee of Clinical Research. All participants gave written informed consent.

Participants and diagnostic criteria Between 2000 and 2007, we recruited 3,612 persons fulfilling the inclusion/ exclusion criteria, with 3,344 (1,718 men and 1,626 women, $52.6 \pm 14.5[$ mean $\pm \mathrm{SD}]$ years of age) providing all required information for study. The remaining 268 individuals were excluded due to inadequate ABPM sampling at baseline and non-consent for additional ABPM evaluations. At the time of recruitment, 688 randomised hypertensive patients already had a diagnosis of type 2 diabetes and, therefore, were excluded from the analyses reported here. Among the remaining 2,656 participants, 644 were normotensive at baseline and, accordingly, were not participants in any aspect of the trial involving randomisation to a treatment-time for ingestion of hypertension medications. The final evaluated population for the hypotheses tested herein thus consisted of 2,012 hypertensive patients without diabetes -976 men and 1,036 women, $52.7 \pm 13.6$ years of age (Fig. 1).

Hypertension in untreated participants was defined according to current ABPM criteria, i.e., awake systolic (SBP)/diastolic (DBP) BP mean $\geq 135 / 85 \mathrm{mmHg}$ or asleep SBP/DBP mean $\geq 120 / 70 \mathrm{mmHg}[27,28]$. New-onset diabetes was defined as fasting glucose $\geq 7.0 \mathrm{mmol} / \mathrm{l}$ on at least two clinical assessments $\geq 3$ months apart [29]; none of the participants received any glucose-lowering treatment during the course of the study. CKD was defined as either an estimated GFR $<60 \mathrm{ml} \mathrm{min}^{-1} 1.73 \mathrm{~m}^{-2}$, albuminuria (urinary albumin excretion $\geq 30 \mathrm{mg} / 24 \mathrm{~h}$ urine), or both, on at least two occasions $\geq 3$ months apart [30]. GFR ( $\left.\mathrm{ml} \mathrm{min}^{-1} 1.73 \mathrm{~m}^{-2}\right)$ was estimated by the CKD Epidemiology Collaboration (CKD-EPI) equation [31]. Diagnosis of the metabolic syndrome was established by the National Cholesterol Education Program Adult Treatment Panel III revised definition [32].

Study design The MAPEC study was a prospective, randomised, open-label, blinded endpoint (PROBE) trial. A major goal of the study was to assess the effect of treatmenttime regimen of prescribed BP-lowering medications on CVD, new-onset diabetes and CKD outcomes. Thus, patients were randomised either to ingest all BP-lowering medications upon awakening or the complete daily dose of $\geq 1$ of them at bedtime and the remaining ones (if any) upon awakening. Assignment of participants to the two treatment-time regimens was done according to the order of recruitment, following an allocation table constructed by a computerised randomnumber generator.

The study did not specify or require one unique investigational hypertension medication; rather, participating physicians were given the choice of prescribing, as first-line therapy in previously untreated patients, one medication of any of the recommended therapeutic classes [28]. Randomisation of patients to treatment-time (awakening or bedtime) was done separately for each allowed initial individual hypertension monotherapy (valsartan, telmisartan, olmesartan, ramipril, spirapril, amlodipine, nifedipine GITS, nebivolol, torasemide, doxazosin GITS) to ensure the proportion of patients treated with each medication was similar across the morning- and bedtime-treatment arms. If based on ABPM threshold criteria, the $\mathrm{ABP}$ of a given patient remained uncontrolled at any time during follow-up (see below), additional medications could be added in keeping with current clinical practice.

Participants with resistant hypertension were randomised either to: (1) modification of the nature of their treatment by exchanging one hypertension medication with a new one, and thus without alteration of the total number of medications, but retaining the upon-waking ingestion time for all of them (awakening treatment regimen); or (2) the same exchange of one medication with a new one, but prescribing its ingestion at bedtime instead of morning (bedtime-treatment regimen) [21, 24]. If, during follow-up, ABP remained uncontrolled, investigators were permitted to: (1) exchange additional hypertension medications for others of different classes (keeping always the upon-waking ingestion time schedule of all medications) in patients of the awakening treatment regimen group; (2) progressively shift additional hypertension medications (that were being ingested upon awakening) to bedtime in patients of the bedtime-treatment regimen group; or (3) prescribe additional BP-lowering medications in patients of either group.

Changes in therapeutic scheme during follow-up in uncontrolled patients (those with ABP above the thresholds provided above) were always based on the results from periodic evaluation by ABPM. Adherence to the time-of-day (awakening or bedtime) schedule of treatment and prescribed medication(s) was enforced, although not measured, at each followup visit. Adverse events, including type, duration, seriousness, intensity and possible relation to hypertension treatment, were registered as spontaneously reported by the patient and/or detected by the investigators through non-directive questioning and physical examination.

$\mathrm{ABP}$, wrist activity and other assessments At inclusion and at every scheduled visit for ABPM during follow-up, the SBP and DBP of each participant were automatically measured every 20 min between 07:00 and 23:00 hours and every 30 min during the night for 48 consecutive hours with a calibrated SpaceLabs 90207 ABPM monitor (SpaceLabs, 
Fig. 1 Flow diagram of participants in the study

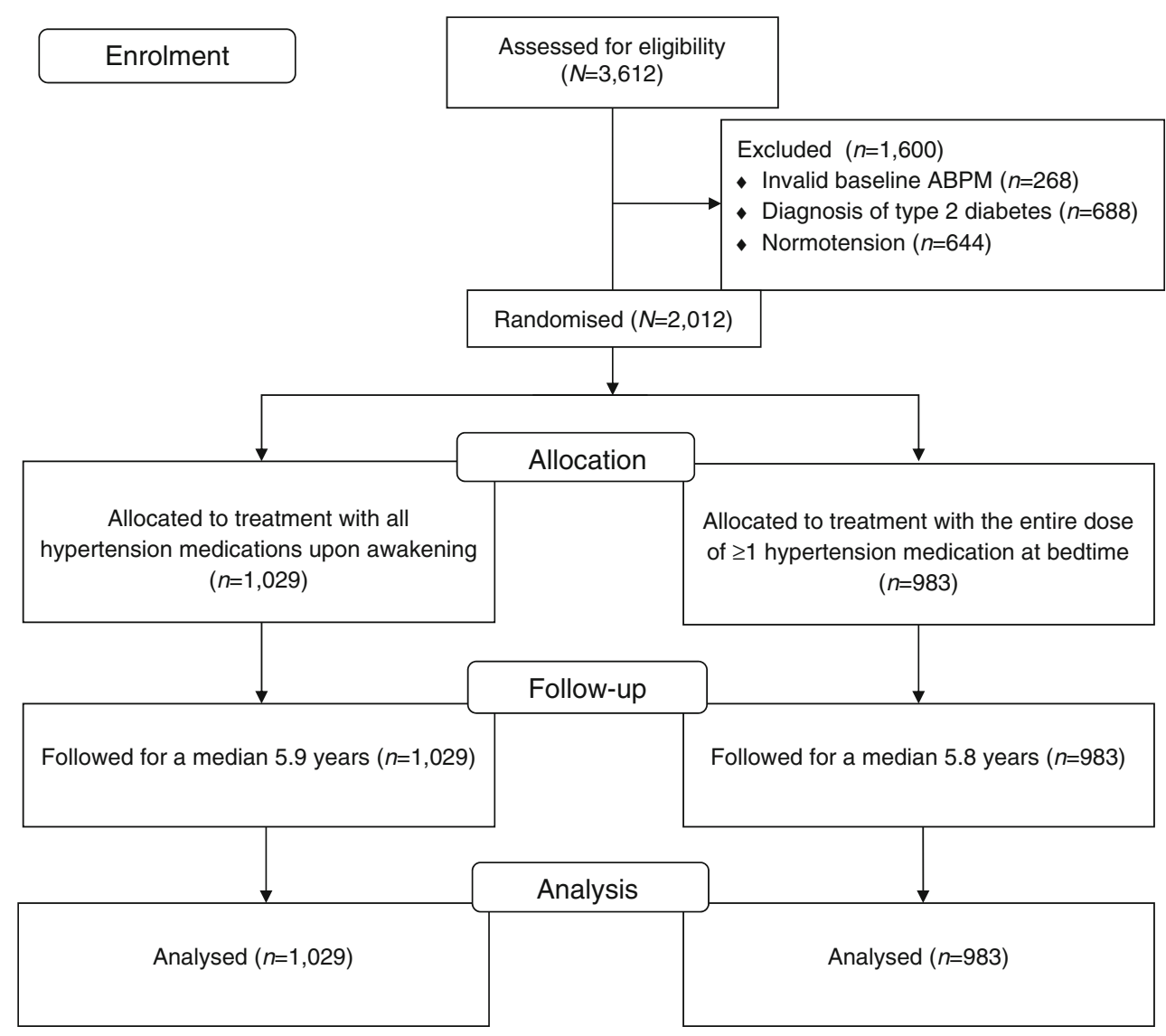

Issaquah, WA, USA). A $48 \mathrm{~h}$, instead of the most common $24 \mathrm{~h}$, monitoring span was chosen to improve reproducibility of results, as accurate calculation of ABP characteristics (including mean BP values) and dipping classification depends markedly on the duration of ABPM [33]. Participants were instructed to adhere to their usual activities with minimal restrictions but to keep a similar activity-rest schedule and avoid daytime napping during the two consecutive days of ABPM. In keeping with current recommendations [27], BP series were considered invalid for analysis, and thus ABPM repeated within the same week, if $\geq 30 \%$ of the measurements were missing, if data were lacking for an interval of $>2 \mathrm{~h}$, if data were obtained when the rest-activity schedule was irregular during the 2 days of monitoring, or if the night-time sleep period was $<6 \mathrm{~h}$ or $>12 \mathrm{~h}$.

All participants also wore an actigraph (Mini-Motion-Logger, Ambulatory Monitoring, Ardsley, NY, USA) on the dominant wrist to record the level of physical activity at 1-min intervals during each $48 \mathrm{~h}$ ABPM. Actigraphy data were used to verify absence of daytime napping and to precisely define the commencement and termination of the daytime awake and night-time asleep spans of each participant using dedicated software [34] to accurately derive the respective ABP means.

Blood withdrawal from an antecubital vein was carried out in the clinic between 08:00 and 09:00 hours, after overnight fasting, the same week when each $48 \mathrm{~h}$ ABPM session was initiated. The patients collected their urine during the first $24 \mathrm{~h}$ of ABPM. Blood and urine samples were analysed using routine automatic techniques in the hospital laboratory. Just before commencing each $48 \mathrm{~h}$ ABPM session, the same investigator obtained six consecutive clinic BP measurements using a validated automatic oscillometric device (HEM-705IT, Omron Health Care, Vernon Hills, IL, USA) after the participant had rested in a seated position for $\geq 10 \mathrm{~min}$.

Follow-up Participants underwent the same evaluation procedure described above, including conventional daytime clinic $\mathrm{BP}$ measurement, $48 \mathrm{~h} \mathrm{ABPM}$ /wrist activity monitoring and blood/urine analysis, annually or more frequently (3 months after any investigator-ordered change in therapy to improve ABP control). Investigators blinded to the hypertension treatment scheme of the patients and not involved in clinic evaluations, ABP measurement and statistical analyses assessed the development of new-onset diabetes, as defined above, among other outcome variables of interest. Complete clinical records of all enrolled participants, including all periodic laboratory tests performed during follow-up, were reviewed at least annually plus the year following the last ABPM. 
Statistical methods ABPM profiles were edited according to conventional criteria to correct for measurement errors and outliers; SBP readings $>250$ or $<70 \mathrm{mmHg}, \mathrm{DBP}>150$ or $<40 \mathrm{mmHg}$ and pulse pressure (PP, SBP minus DBP) $>150$ or $<20 \mathrm{mmHg}$ were automatically discarded. The ' $48 \mathrm{~h} \mathrm{ABP}$ mean' was calculated as the average of all valid readings obtained throughout the $48 \mathrm{~h}$ assessment span. Awake and asleep ABP means were calculated from the $48 \mathrm{~h}$ monitoring as the average of all valid readings obtained during the hours of daytime activity or night-time sleep, respectively, as differentiated by wrist actigraphy. Sleep-time relative BP decline (index of BP dipping), defined as \% decrease in mean BP during night-time sleep relative to mean BP during daytime activity, was calculated as: [(awake ABP mean-asleep ABP mean)/ awake ABP mean] $\times 100$, incorporating all the data sampled by $48 \mathrm{~h}$ ABPM. For comparative purposes, a patient was defined as dipper if the sleep-time relative SBP decline was $\geq 10 \%$, and as non-dipper otherwise $[27,28]$.

Demographic and clinical characteristics were compared on an intention-to-treat basis among participants randomised to the two treatment-time regimens-(1) all hypertension medications ingested upon awakening or (2) the entire daily dose of $\geq 1$ BP-lowering medications ingested at bedtime- - by two-sided $t$ test (continuous variables) or nonparametric $\chi^{2}$ test (proportions). The Cox proportional-hazard model, adjusted for significant confounding variables, was used to estimate $\mathrm{HR}$, with $95 \% \mathrm{CI}$, for events associated with treatmenttime regimen and class of hypertension medication. For survival analysis, follow-up was established as either the time to the confirmed diagnosis of diabetes or the time to the last clinical evaluation in non-event participants. Survival curves were generated using the Kaplan-Meier product-limit method and compared by the Mantel logrank test.

\section{Results}

Demographic characteristics, laboratory variables, and ABP of morning- and bedtime-treatment groups Among the 2,012 participants, 1,029 were randomised to the morning and 983 to the bedtime-treatment regimen, with a median follow-up of 5.9 and 5.8 years, respectively. At baseline, the two groups were equivalent for the prevalence of the metabolic syndrome, obstructive sleep apnoea, anaemia, CKD and obesity, plus all evaluated anthropometric variables (Table 1). Clinic BP, average ABP values and prevalence of non-dipping at baseline were also equivalent between the two groups (Table 1).

In keeping with the study design, there were no differences in the classes and number of hypertension medications used for therapy between the patients of the two treatment-time groups (Table 2). The proportion of patients in the morning and bedtime-therapy groups treated with statins $(16.2 \%$ vs
$15.3 \%$, respectively; $p=0.551)$ or low-dose (100 mg/day) aspirin $(10.5 \%$ vs $11.4 \% ; p=0.519)$ was also similar.

The data of the last evaluation revealed significantly lower asleep SBP/DBP means in participants randomised to the bedtime- than morning-treatment regimen $(p<0.001$; Table 2$)$. The sleep-time relative SBP/DBP decline was significantly greater and, accordingly, the proportion of patients with the non-dipper BP pattern significantly lower, in the bedtimethan the morning-treatment regimen group (32\% vs $52 \%$; $p<0.001)$. The proportion of participants with properly controlled ABP, mainly during night-time sleep, was also significantly greater among those treated at bedtime $(p<0.001$; Table 2). There were no treatment-time differences in the prevalence of patients reporting adverse effects $(6.0 \%$ vs $6.3 \%$ for morning- and bedtime-treatment regimen, respectively; $p=0.569$ ). Due to strict patient control by periodic ABPM during follow-up [21], only two and three patients in the morning- and bedtime-treatment groups, respectively, had sleep-time hypotension, as defined by current ABPM criteria [27], at their final evaluation; none of them developed newonset diabetes.

New-onset diabetes risk according to hypertension treatment-time regimen During the median follow-up period of 5.9 years (range 1.0-8.9 years), 171 participants developed type 2 diabetes. Patients ingesting $\geq 1$ hypertension medications at bedtime showed a significantly lower HR of newonset diabetes (adjusted by the only significant influential characteristics, among all variables listed in Table 1, of fasting glucose, waist circumference, asleep BP mean, dipping classification and $\mathrm{CKD}$ ) than those ingesting all medications upon awakening (unadjusted HR 0.41 [95\% CI 0.29, 0.58]; adjusted HR 0.43 [0.31, 0.61]; event-rate $4.8 \%$ vs $12.1 \%$ in the bedtime- and morning-treatment groups; $p<0.001)$. Independent of hypertension treatment-time, increased HR of newonset diabetes was jointly associated with elevated fasting glucose $(3.11$ [2.43, 3.98], $p<0.001$, per mmol/1); larger waist circumference (1.03 [1.02, 1.04], $p<0.001$, per cm); greater asleep BP (1.12 [1.06, 1.17], $p<0.001$ per $5 \mathrm{mmHg})$; nondipping $(1.45[1.03,2.05], p=0.027$, compared with dipper BP pattern); and presence of CKD (1.41 [1.02, 1.95], $p=$ 0.039 , compared with absence of CKD). Figure 2 presents the Kaplan-Meier survival curves for the patients of the two treatment-time groups, documenting the highly significant difference between treatment-time groups in new-onset diabetes event-free survival (logrank 28.0, $p<0.001$ ).

New-onset diabetes risk according to class of hypertension medication and treatment-time regimen Figure $3 \mathrm{a}$ shows the HR of new-onset diabetes for participants of the morning-treatment regimen group categorised according to class of hypertension medication ingested upon awakening. Each category includes all patients ingesting that particular 
Table 1 Baseline characteristics of patients investigated categorised according to treatment-time regimen (either all hypertension medications upon awakening or the entire dose of $\geq 1$ medications at bedtime)

\begin{tabular}{|c|c|c|c|}
\hline Variable & Awakening & Bedtime & $p$ between groups \\
\hline \multicolumn{4}{|l|}{ Demographic characteristics } \\
\hline Patients $(n)$ & 1,029 & 983 & \\
\hline Sex $(\%$ men $)$ & 48.0 & 49.0 & 0.645 \\
\hline The metabolic syndrome $(\%)^{\mathrm{a}}$ & 50.8 & 46.7 & 0.277 \\
\hline Cigarette smoking (\%) & 15.1 & 14.6 & 0.739 \\
\hline Obesity $(\%)^{\mathrm{b}}$ & 38.4 & 38.9 & 0.827 \\
\hline Obstructive sleep apnoea (\%) & 8.7 & 8.4 & 0.824 \\
\hline Anaemia (\%) & 6.4 & 6.1 & 0.774 \\
\hline $\operatorname{CKD}(\%)^{\mathrm{c}}$ & 22.4 & 21.3 & 0.554 \\
\hline Previous CVD events (\%) & 6.8 & 6.3 & 0.674 \\
\hline Duration of known hypertension (years) & $6.8 \pm 8.0$ & $6.7 \pm 7.8$ & 0.652 \\
\hline \multicolumn{4}{|l|}{ Anthropometric variables and office BP } \\
\hline Age (years) & $53.2 \pm 13.8$ & $52.2 \pm 13.4$ & 0.191 \\
\hline Height $(\mathrm{cm})$ & $162.1 \pm 9.9$ & $162.5 \pm 9.9$ & 0.332 \\
\hline Weight (kg) & $77.3 \pm 15.3$ & $77.4 \pm 14.8$ & 0.942 \\
\hline BMI $\left(\mathrm{kg} / \mathrm{m}^{2}\right)$ & $29.4 \pm 4.9$ & $29.2 \pm 4.6$ & 0.520 \\
\hline Waist (cm) & $95.0 \pm 12.3$ & $94.5 \pm 11.5$ & 0.288 \\
\hline Clinic SBP $(\mathrm{mmHg})^{\mathrm{d}}$ & $152.9 \pm 18.5$ & $153.4 \pm 18.5$ & 0.423 \\
\hline Clinic DBP $(\mathrm{mmHg})^{\mathrm{d}}$ & $88.3 \pm 10.6$ & $89.0 \pm 10.8$ & 0.123 \\
\hline Clinic PP $(\mathrm{mmHg})^{\mathrm{d}}$ & $64.6 \pm 13.7$ & $64.4 \pm 13.4$ & 0.485 \\
\hline Clinic heart rate (beats $/ \mathrm{min})^{\mathrm{d}}$ & $74.1 \pm 12.2$ & $75.0 \pm 12.8$ & 0.189 \\
\hline \multicolumn{4}{|l|}{ Clinical laboratory test values } \\
\hline Glucose (mmol/l) & $5.43 \pm 0.64$ & $5.42 \pm 0.65$ & 0.604 \\
\hline Creatinine $(\mu \mathrm{mol} / \mathrm{l})$ & $84.0 \pm 20.3$ & $84.8 \pm 18.5$ & 0.861 \\
\hline Uric acid $(\mu \mathrm{mol} / \mathrm{l})$ & $339.0 \pm 95.2$ & $339.0 \pm 89.2$ & 0.316 \\
\hline Total cholesterol (mmol/l) & $5.52 \pm 0.96$ & $5.54 \pm 1.04$ & 0.667 \\
\hline Triacylglycerol (mmol/l) & $1.32 \pm 0.92$ & $1.28 \pm 0.73$ & 0.326 \\
\hline HDL-cholesterol (mmol/l) & $1.27 \pm 0.40$ & $1.26 \pm 0.40$ & 0.606 \\
\hline LDL-cholesterol (mmol/l) & $3.64 \pm 0.84$ & $3.67 \pm 0.89$ & 0.507 \\
\hline Haemoglobin (g/l) & $142 \pm 14$ & $143 \pm 13$ & 0.640 \\
\hline Fibrinogen $(\mu \mathrm{mol} / \mathrm{l})$ & $9.40 \pm 2.32$ & $9.29 \pm 2.20$ & 0.323 \\
\hline Erythrocyte sedimentation rate $(\mathrm{mm} / \mathrm{h})$ & $14.0 \pm 10.9$ & $13.7 \pm 11.2$ & 0.621 \\
\hline Estimated GFR & $80.0 \pm 19.5$ & $79.9 \pm 16.5$ & 0.969 \\
\hline \multicolumn{4}{|l|}{$\mathrm{ABP}$} \\
\hline Duration of nocturnal rest (h) & $8.9 \pm 1.4$ & $8.9 \pm 1.3$ & 0.271 \\
\hline Awake SBP mean (mmHg) & $134.2 \pm 14.2$ & $134.5 \pm 13.2$ & 0.543 \\
\hline Asleep SBP mean (mmHg) & $120.0 \pm 14.3$ & $120.4 \pm 14.0$ & 0.459 \\
\hline 48 h SBP mean (mmHg) & $129.7 \pm 13.6$ & $129.9 \pm 12.8$ & 0.866 \\
\hline Sleep-time relative SBP decline $(\%)^{\mathrm{e}}$ & $10.4 \pm 7.1$ & $10.4 \pm 7.4$ & 0.845 \\
\hline Awake DBP mean (mmHg) & $84.0 \pm 10.8$ & $84.6 \pm 10.4$ & 0.289 \\
\hline Asleep DBP mean (mmHg) & $70.7 \pm 9.8$ & $70.9 \pm 9.8$ & 0.690 \\
\hline 48 h DBP mean (mmHg) & $79.8 \pm 10.1$ & $80.4 \pm 9.8$ & 0.278 \\
\hline Sleep-time relative DBP decline (\%) ${ }^{\mathrm{e}}$ & $15.6 \pm 8.0$ & $16.0 \pm 7.9$ & 0.161 \\
\hline Non-dipper $(\%)^{\mathrm{f}}$ & 45.1 & 44.2 & 0.671 \\
\hline
\end{tabular}

Values are shown as mean $\pm \mathrm{SD}$, unless otherwise indicated

${ }^{a}$ The metabolic syndrome: National Cholesterol Education Program Adult Treatment Panel III revised definition [32]

${ }^{\mathrm{b}}$ Obesity: BMI $\geq 30 \mathrm{~kg} / \mathrm{m}^{2}$

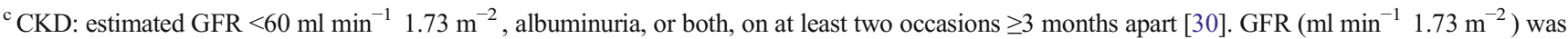
estimated using the CKD-EPI equation [31]

${ }^{\mathrm{d}}$ Values correspond to the average of six conventional BP measurements obtained for each participant at the clinic before starting $48 \mathrm{~h}$ ABPM

${ }^{\mathrm{e}}$ Sleep-time relative BP decline, index of BP dipping, is defined as \% decline in mean BP during night-time sleep relative to mean BP during daytime activity, and calculated as: ([awake BP mean-asleep BP mean]/awake BP mean) $\times 100$

${ }^{\mathrm{f}}$ Non-dipper: patients with sleep-time relative SBP decline $<10 \%$, using data sampled by ABPM for 48 consecutive hours 
Table 2 Final characteristics of patients investigated categorised according to treatment-time regimen (either all hypertension medications upon awakening or the entire dose of $\geq 1$ medications at bedtime)

\begin{tabular}{|c|c|c|c|}
\hline Variable & Awakening & Bedtime & $p$ between groups \\
\hline Patients $(n)$ & 1,029 & 983 & \\
\hline \multicolumn{4}{|l|}{ Hypertension treatment } \\
\hline No. of medications & $2.0 \pm 1.1$ & $2.0 \pm 1.0$ & 0.310 \\
\hline 1 medication $(\%)$ & 48.9 & 46.3 & 0.244 \\
\hline 2 medications $(\%)$ & 20.1 & 22.2 & 0.258 \\
\hline$\geq 3$ medications $(\%)$ & 31.0 & 31.5 & 0.796 \\
\hline ARB $(\%)$ & 62.1 & 60.7 & 0.529 \\
\hline ACEI $(\%)$ & 21.0 & 19.4 & 0.384 \\
\hline CCB $(\%)$ & 35.2 & 37.7 & 0.233 \\
\hline$\alpha$-blocker $(\%)$ & 21.4 & 22.4 & 0.587 \\
\hline$\beta$-blocker $(\%)$ & 20.5 & 22.1 & 0.390 \\
\hline Diuretic (\%) & 36.7 & 36.0 & 0.736 \\
\hline \multicolumn{4}{|l|}{ Clinic and ambulatory BP } \\
\hline Clinic SBP $(\mathrm{mmHg})^{\mathrm{a}}$ & $143.6 \pm 20.9$ & $141.6 \pm 19.3$ & 0.022 \\
\hline Clinic DBP $(\mathrm{mmHg})^{\mathrm{a}}$ & $82.7 \pm 12.4$ & $82.6 \pm 11.7$ & 0.890 \\
\hline Clinic PP $(\mathrm{mmHg})^{\mathrm{a}}$ & $60.9 \pm 14.8$ & $59.0 \pm 13.3$ & 0.002 \\
\hline Clinic heart rate (beats $/ \mathrm{min})^{\mathrm{a}}$ & $72.6 \pm 13.1$ & $73.2 \pm 13.3$ & 0.344 \\
\hline Awake SBP mean $(\mathrm{mmHg})^{\mathrm{b}}$ & $125.9 \pm 14.1$ & $125.6 \pm 13.2$ & 0.644 \\
\hline Asleep SBP mean $(\mathrm{mmHg})^{\mathrm{c}}$ & $114.4 \pm 15.2$ & $109.6 \pm 13.9$ & $<0.001$ \\
\hline $48 \mathrm{~h} \mathrm{SBP}$ mean $(\mathrm{mmHg})$ & $122.3 \pm 13.7$ & $120.3 \pm 12.8$ & $<0.001$ \\
\hline Sleep-time relative SBP decline $(\%)^{\mathrm{d}}$ & $9.0 \pm 8.3$ & $12.6 \pm 7.2$ & $<0.001$ \\
\hline Awake DBP mean $(\mathrm{mmHg})^{\mathrm{b}}$ & $77.6 \pm 10.5$ & $78.2 \pm 10.1$ & 0.202 \\
\hline Asleep DBP mean $(\mathrm{mmHg})^{\mathrm{c}}$ & $66.3 \pm 10.5$ & $63.4 \pm 9.8$ & $<0.001$ \\
\hline 48 h DBP mean $(\mathrm{mmHg})$ & $74.0 \pm 10.0$ & $73.2 \pm 9.5$ & 0.080 \\
\hline Sleep-time relative DBP decline $(\%)^{\mathrm{d}}$ & $14.3 \pm 10.0$ & $18.7 \pm 8.5$ & $<0.001$ \\
\hline Non-dipper $(\%)^{\mathrm{e}}$ & 52.0 & 31.7 & $<0.001$ \\
\hline Controlled ABP $(\%)^{\mathrm{f}}$ & 48.8 & 58.2 & $<0.001$ \\
\hline Controlled awake BP (\%) & 68.8 & 71.0 & 0.282 \\
\hline Controlled asleep BP (\%) & 59.1 & 71.6 & $<0.001$ \\
\hline
\end{tabular}

Values are shown as mean $\pm \mathrm{SD}$, unless otherwise indicated

${ }^{a}$ Values correspond to the average of six conventional BP measurements obtained for each participant at the clinic before starting $48 \mathrm{~h}$ ABPM

${ }^{\mathrm{b}}$ Awake SBP/DBP mean was considered controlled if $<135 / 85 \mathrm{mmHg}$

${ }^{\mathrm{c}}$ Asleep SBP/DBP mean was considered controlled if $<120 / 70 \mathrm{mmHg}$

${ }^{\mathrm{d}}$ Sleep-time relative BP decline, index of BP dipping, is defined as \% decline in mean BP during night-time sleep relative to mean BP during daytime activity, and calculated as: ([awake BP mean-asleep BP mean]/awake BP mean) $\times 100$

${ }^{\mathrm{e}}$ Non-dipper: patients with sleep-time relative SBP decline $<10 \%$, using data sampled by ABPM for 48 consecutive hours

${ }^{\mathrm{f}} \mathrm{ABP}$ was considered controlled if both awake and asleep SBP/DBP means were below the corresponding thresholds provided above class of hypertension medication upon awakening, either alone or along with other classes of medications also ingested upon awakening. We used as the reference group patients ingesting an ARB, as this category had the lowest new-onset diabetes event-rate compared with all the other hypertension medications classes. The adjusted HR of new-onset diabetes was fully equivalent across all classes of medications when ingested in the morning.
Figure $3 \mathrm{~b}$ presents the adjusted HR of new-onset diabetes for participants of the bedtime-treatment regimen group. Each category includes patients who were ingesting the entire daily dose of that particular class of hypertension medication at bedtime, either alone or along with other hypertensive medications ingested as entire daily doses also at bedtime or upon awakening, in keeping with the design of the trial. Patients ingesting an ARB, ACEI or $\beta$-blocker (primarily nebivolol) 


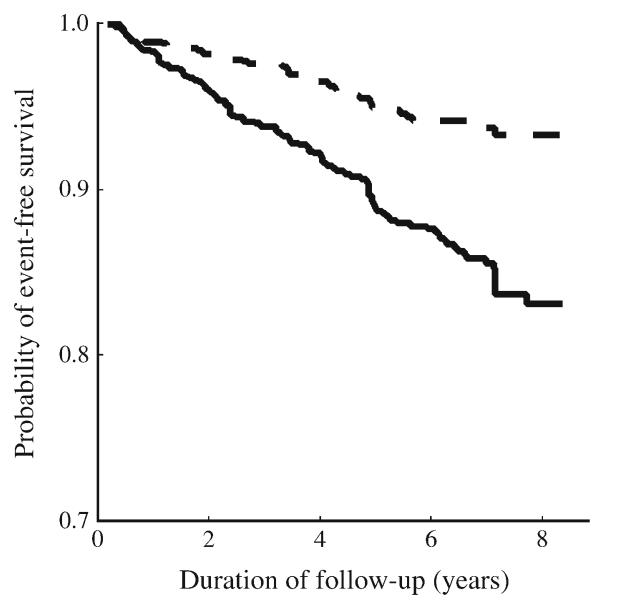

Patients at risk

$\begin{array}{lrrrrr}\text { Awakening } & 1,029 & 978 & 756 & 543 & 309 \\ \text { Bedtime } & 983 & 965 & 740 & 514 & 286\end{array}$

Fig. 2 Kaplan-Meier survival curves for new-onset diabetes as a function of time-of-day of hypertension treatment, i.e. for hypertensive patients ingesting either all their prescribed BP-lowering medications upon awakening (black line) or the entire daily dose of $\geq 1$ of them at bedtime (dashed line). Logrank 28.0, $p<0.001$

at bedtime had significantly lower HR of new-onset diabetes than patients ingesting any other medication class also at bedtime (Fig. 3b).

We additionally compared the risk of new-onset diabetes between patients of the two treatment-time regimen groups further categorised as a function of the class of hypertensive medication used for therapy. Figure 4 shows the HR of new-

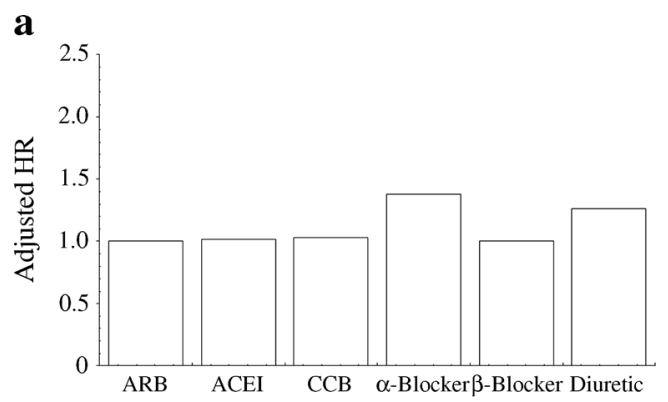

b

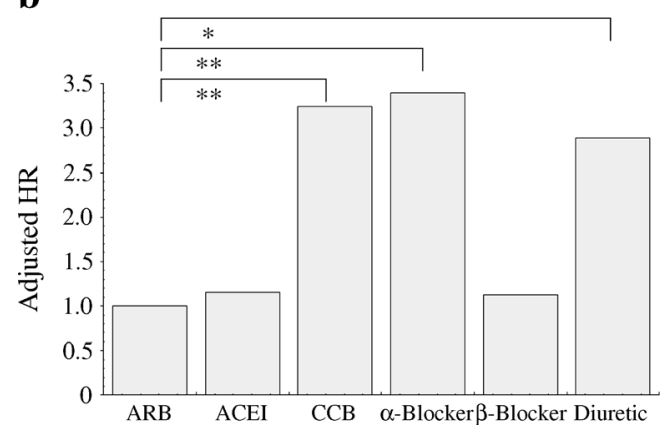

Fig. 3 Adjusted HR of new-onset diabetes as a function of class of hypertension medication included in the therapeutic scheme of hypertensive patients ingesting all hypertension medications upon awakening (a) or the entire dose of $\geq 1$ of them at bedtime (b). ${ }^{*} p<0.05,{ }^{* *} p<0.01$

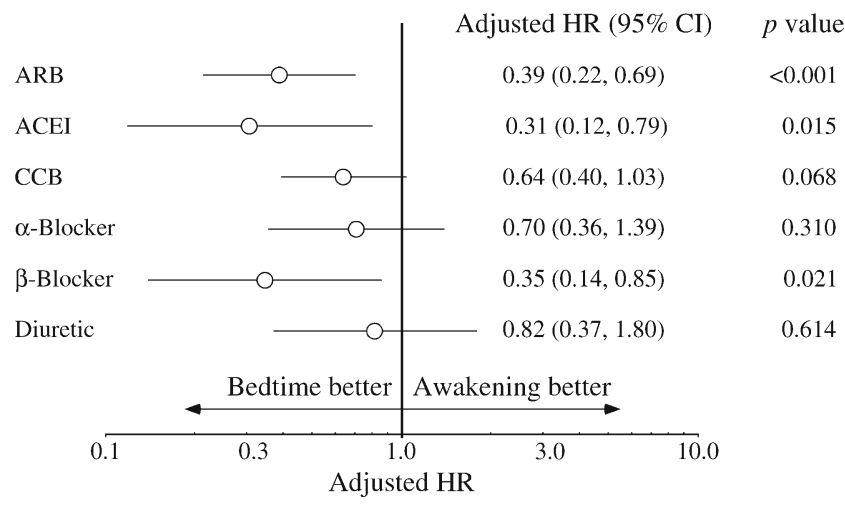

Fig. 4 Adjusted HR (with 95\% CI) of new-onset diabetes as a function of medication class and hypertension treatment-time regimen for hypertensive patients, i.e., those ingesting either all medications upon awakening or the entire dose of $\geq 1$ of them at bedtime

onset diabetes for participants ingesting any given class of hypertension medication upon awakening vs at bedtime. Comparison for any given class was done between patients ingesting the tested class of medication upon awakening either alone or along with other medications ingested also on awakening, vs participants ingesting the same specified class of medication at bedtime. Significant benefit in terms of reduction of the risk of new-onset diabetes was observed for bedtime compared with awakening treatment with ARBs (HR 0.39 [0.22, 0.69]; $p<0.001)$, ACEIs $(0.31$ [0.12, 0.79], $p=0.015)$ and $\beta$-blockers (0.35 [0.14, 0.85], $p=0.021)$, but not for medications of the other three classes (CCBs, $\alpha$ blockers and diuretics) of hypertension therapies (Fig. 4).

\section{Discussion}

The MAPEC study is the first, and thus far only, reported trial to assess prospectively the hypothesis that bedtime treatment with the entire daily dose of $\geq 1$ hypertension medications exerts not only better BP control but better protection against new-onset diabetes than conventional therapy with all such medications ingested upon awakening. The results document, first, greater $\mathrm{ABP}$ control in hypertensive patients randomised to the bedtime-treatment group. The main differences between the bedtime vs morning-time treatment groups in terms of ABP control were achievement in the former of: (1) significantly lower asleep BP mean; and (2) greater sleep-time relative BP decline, without loss of awake BP-lowering efficacy (Table 2). The ingestion-time-dependent effects on asleep BP control were strongly associated with attenuated risk of newonset diabetes. Indeed, in the MAPEC study the progressive decrease in the asleep BP mean during follow-up was the most significant predictor of reduced risk of new-onset diabetes, independent of other conventional variables associated with increased risk such as older age, larger waist circumference, elevated fasting glucose, and CKD diagnosis [20]. As 
documented in a series of prospective controlled trials extensively reviewed elsewhere $[7,8]$, bedtime hypertension treatment is the most cost-effective and simplest strategy of successfully achieving the therapeutic goal of adequate asleep BP reduction and control. One could thus conclude that the significant $57 \%$ reduction in the risk of new-onset diabetes by ingestion of the entire daily dose of $\geq 1$ BP-lowering medications at bedtime, compared with ingestion of all such medications upon-waking (Fig. 2), is linked to better achievement of this novel hypertension therapeutic goal through therapeutic targeting of the underlying biological mechanisms. The total new-onset diabetes event-rate was somehow intermediate between that reported in the HOPE [5] and DREAM trials [6]; both of these trials had a lower follow-up than MAPEC, but in DREAM was restricted to patients with impaired fasting glucose and thus at higher risk for new-onset diabetes.

The randomisation for this trial, independently done for each of the allowed choices of hypertension medications, and the so-far unique approach of systematic periodic (at least annual) patient evaluation by highly reproducible $48 \mathrm{~h}$ ABPM to guide required changes in therapy, allowed prospective comparative investigation of the influence of different classes of hypertension medications on the risk of new-onset diabetes. The findings summarised in Figs 3, 4 document significant differences in risk of new-onset diabetes between individual classes of hypertension medications when treatment-time is taken into consideration. The adjusted HR of new-onset diabetes across all six classes of the tested hypertension medications was equivalent in those patients randomised to ingest all of them as full daily doses upon awakening (Fig. 3a). However, among patients randomised to the bedtime regimen, ingestion of an ARB, ACEI or $\beta$-blocker was associated with significantly lower HR of new-onset diabetes compared with ingestion of any other class of medication also at bedtime. We wish to emphasise that nebivolol was the $\beta$-blocker most participants were treated with, in fact, the only one allowed for first randomisation among untreated patients. Nebivolol is a third generation, long acting $\beta$-blocker medication that exerts endothelium-dependent vasodilation through activation of the L-arginine/nitric oxide pathway, significant reduction of glucose and lipids in patients with diabetes [35], and regulatory effect on the RAAS [36]. Accordingly, our findings might not be extrapolated to all $\beta$-blockers as a class, an issue that requires future investigation. With these considerations notwithstanding, the results depicted in Fig. 4 indicate that reduced risk of new-onset diabetes is significantly associated with RAAS blockade therapy ingested at bedtime. Since the RAAS activates during night-time sleep [9], the results also suggests, in keeping with the hypotheses for this trial, that bedtime, rather than upon awakening, RAAS inhibition or blockade and the corresponding potential improved control of impaired glucose and insulin tolerance might be among the factors associated with reduced risk of new-onset diabetes.
Our study has some potential limitations. First, sample size of the single-centre MAPEC study is limited to evaluate the potential predictive value of different hypertension medications within each therapeutic class. Second, the reported findings require validation in other ethnic groups. Finally, the use of a PROBE design might also be considered a limitation; however, the PROBE design was specifically developed for the conduct of long-term outcome trials, though it is also frequently used for the conduct of short-term efficacy clinical studies in patients evaluated in a blinded manner by ABPM. The design of the MAPEC Study also has several strengths [21], mainly being the first trial yet to: (1) provide results based on systematic periodic, at least annual, multiple evaluations by ABPM and wrist actigraphy throughout the median 5.9 years of follow-up; (2) define hypertension as an inclusion criterion based solely on ABP measurements; (3) prescribe changes in therapeutic intervention during follow-up to improve control of ABP, instead of daytime clinic BP; and (4) randomise patients either to ingest all BP-lowering medications upon awakening or the complete daily dose of $\geq 1$ of them at bedtime, thus according to the individualised rest/activity cycle that synchronises the documented predictable-in-time $24 \mathrm{~h}$ changes in RAAS activation and BP patterning [9].

In conclusion, according to this prospective evaluation, in hypertensive patients without diabetes, ingestion of the entire daily dose of $\geq 1$ BP-lowering medications at bedtime compared with ingestion of all such medications upon-awakening, results in significantly improved asleep ABP control and prevention of new-onset diabetes. Moreover, the safety of bedtime- and morning-treatment regimens was similar, a finding consistent with previous publications reporting bedtime compared with morning BP therapy significantly improves ABP reduction without any increase in adverse effects [37]. Finally, antagonism of the RAAS by reduction of angiotensin II formation and blockade of its receptors achieved either by a bedtime ACEI or ARB ingestion strategy is superior to any other treatment strategy for reducing risk of new-onset diabetes. Future prospective intervention trials that incorporate periodic, annually or more frequent, ABPM assessments during long-term follow-up evaluation, as done in the MAPEC study and the currently ongoing multicentre Hygia project [38, 39], are necessary to confirm the reduced risk of new-onset diabetes by bedtime hypertension chronotherapy.

Duality of interest The authors declare that there is no duality of interest associated with this manuscript.

Contribution statement RCH and DEA contributed to every aspect of this article, including conception and design of the study, acquisition and interpretation of the data, and drafting the manuscript. AM and JRF contributed to study design, discussion, statistical analyses and revision of the manuscript. All listed authors approved the final version of the 
manuscript. $\mathrm{RCH}$ is the guarantor of this work. The authors report no potential conflicts of interest relevant to this article.

Funding This independent investigator-promoted research was supported by unrestricted grants from Ministerio de Ciencia e Innovación (SAF2006-6254-FEDER; SAF2009-7028-FEDER); Xunta de Galicia (PGIDIT03-PXIB-32201PR; INCITE07-PXI-322003ES; INCITE08E1R-322063ES; INCITE09-E2R-322099ES; 09CSA018322PR); and Vicerrectorado de Investigación, University of Vigo.

\section{References}

1. Cooper SA, Whaley-Connell A, Habibi J et al (2007) Reninangiotensin-aldosterone system and oxidative stress in cardiovascular insulin resistance. Am J Physiol Heart Circ Physiol 293:H2009-H2023

2. Gillespie EL, White CM, Kardas M, Lindberg M, Coleman CI (2005) The impact of ACE inhibitors and angiotensin II type 1 receptor blockers on the development of new-onset type 2 diabetes. Diabetes Care 28:2261-2266

3. Putnam K, Shoemaker R, Yiannikouris F, Cassis LA (2012) The renin-angiotensin system: a target of and contributor to dyslipidemias, altered glucose homeostasis, and hypertension of the metabolic syndrome. Am J Physiol Heart Circ Physiol 302:H1219-H1230

4. Yusuf S, Sleight P, Pogue J, Bosch J, Davies R, Dagenais G (2000) Effects of an angiotensin-converting-enzyme inhibitor, ramipril, on cardiovascular events in high-risk patients: the Heart Outcomes Prevention Evaluation Study Investigators. N Engl J Med 342:145-153

5. Yusuf S, Gerstein H, Hoogwerf B et al (2001) Ramipril and the development of diabetes. JAMA 286:1882-1885

6. Trial Investigators DREAM, Bosch J, Yusuf S et al (2006) Effect of ramipril on the incidence of diabetes. N Engl J Med 355:1551-1562

7. Smolensky MH, Hermida RC, Ayala DE, Tiseo R, Portaluppi F (2010) Administration-time-dependent effect of blood pressurelowering medications: basis for the chronotherapy of hypertension. Blood Press Monit 15:173-180

8. Hermida RC, Ayala DE, Fernández JR et al (2013) Administrationtime-differences in effects of hypertension medications on ambulatory blood pressure regulation. Chronobiol Int 30:280-314

9. Portaluppi F, Tiseo R, Smolensky MH, Hermida RC, Ayala DE, Fabbian F (2012) Circadian rhythms and cardiovascular health. Sleep Med Rev 16:151-166

10. Hermida RC, Ayala DE, Fernández JR, Portaluppi F, Fabbian F, Smolensky MH (2011) Circadian rhythms in blood pressure regulation and optimization of hypertension treatment with ACE inhibitor and ARB medications. Am J Hypertens 24:383-391

11. Dolan E, Stanton A, Thijs L et al (2005) Superiority of ambulatory over clinic blood pressure measurement in predicting mortality: the Dublin outcome study. Hypertension 46:156-161

12. Ben-Dov IZ, Kark JD, Ben-Ishay D, Mekler J, Ben-Arie L, Bursztyn M (2007) Predictors of all-cause mortality in clinical ambulatory monitoring. Unique aspects of blood pressure during sleep. Hypertension 49:1235-1241

13. Fagard RH, Celis H, Thijs L et al (2008) Daytime and nighttime blood pressure as predictors of death and cause-specific cardiovascular events in hypertension. Hypertension 51:55-61

14. Hermida RC, Ayala DE, Mojón A, Fernández JR (2011) Decreasing sleep-time blood pressure determined by ambulatory monitoring reduces cardiovascular risk. J Am Coll Cardiol 58:1165-1173
15. Hermida RC, Ayala DE, Fernández JR, Mojón A (2013) Sleep-time blood pressure: prognostic value and relevance as a therapeutic target for cardiovascular risk reduction. Chronobiol Int 30:68-86

16. The ABC-H Investigators, Roush GC, Fagard RH et al (2014) Prognostic impact from clinic, daytime, and nighttime systolic blood pressure in 9 cohorts on 13,844 patients with hypertension. J Hypertens 32:2332-2340

17. Astrup AS, Nielsen FS, Rossing P et al (2007) Predictors of mortality in patients with type 2 diabetes with or without diabetic nephropathy: a follow-up study. J Hypertens 25:2479-2485

18. Bouhanick B, Bongard V, Amar J, Bousquel S, Chamontin B (2008) Prognostic value of nocturnal blood pressure and reversedipping status on the occurrence of cardiovascular events in hypertensive diabetic patients. Diabetes Metab 34:560-567

19. Hermida RC, Ayala DE, Mojón A, Fernández JR (2012) Sleep-time blood pressure as a therapeutic target for cardiovascular risk reduction in type 2 diabetes. Am J Hypertens 25:325-334

20. Hermida RC, Ayala DE, Mojón A, Fernández JR (2015) Sleep-time BP: prognostic marker of type 2 diabetes and therapeutic target for prevention. Diabetologia doi:10.1007/s00125-015-3748-8

21. Hermida RC, Ayala DE, Mojón A, Fernández JR (2010) Influence of circadian time of hypertension treatment on cardiovascular risk: results of the MAPEC study. Chronobiol Int 27:1629-1651

22. Hermida RC, Ayala DE, Mojón A, Fernández JR (2011) Influence of time of day of blood pressure-lowering treatment on cardiovascular risk in hypertensive patients with type 2 diabetes. Diabetes Care 34:1270-1276

23. Hermida RC, Ayala DE, Mojón A, Fernández JR (2011) Bedtime dosing of antihypertensive medications reduces cardiovascular risk in CKD. J Am Soc Nephrol 22:2313-2321

24. Ayala DE, Hermida RC, Mojón A, Fernández JR (2013) Cardiovascular risk of resistant hypertension: dependence on treatment-time regimen of blood pressure-lowering medications. Chronobiol Int 30:340-352

25. Hermida RC, Ayala DE, Mojón A, Fernández JR (2013) Cardiovascular risk of essential hypertension: influence of class, number, and treatment-time regimen of hypertension medications. Chronobiol Int 30:315-327

26. Calhoun DA, Jones D, Textor S et al (2008) Resistant hypertension: diagnosis, evaluation, and treatment. A scientific statement from the American Heart Association Professional Education Committee of the Council for High Blood Pressure Research. Hypertension 51:1403-1419

27. Hermida RC, Smolensky MH, Ayala DE et al (2013) 2013 ambulatory blood pressure monitoring recommendations for the diagnosis of adult hypertension, assessment of cardiovascular and other hypertension-associated risk, and attainment of therapeutic goals. Joint recommendations from the International Society for Chronobiology (ISC), American Association of Medical Chronobiology and Chronotherapeutics (AAMCC), Spanish Society of Applied Chronobiology, Chronotherapy, and Vascular Risk (SECAC), Spanish Society of Atherosclerosis (SEA), and Romanian Society of Internal Medicine (RSIM). Chronobiol Int 30:355-410

28. Mancia G, Fagard R, Narkiewicz K et al (2013) 2013 ESH/ESC guidelines for the management of arterial hypertension: the task force for the management of arterial hypertension of the European Society of Hypertension (ESH) and of the European Society of Cardiology (ESC). J Hypertens 31:1281-1357

29. American Diabetes Association (2013) Standards of medical care in diabetes - 2013. Diabetes Care 36(Suppl 1):S11-S66

30. National Kidney Foundation (2002) K/DOQI clinical practice guidelines on chronic kidney disease: evaluation, classification and stratification. Am J Kidney Dis 39(2 Suppl 1):S1-S266

31. Levey AS, Stevens LA, Schmid CH et al (2009) A new equation to estimate glomerular filtration rate. Ann Intern Med 150:604-612 
32. Grundy SM, Cleeman JI, Daniels SR et al (2005) Diagnosis and management of the metabolic syndrome. An American Heart Association/National Heart, Lung, and Blood Institute Scientific Statement. Circulation 112:2735-2752

33. Hermida RC, Ayala DE, Fontao MJ, Mojón A, Fernández JR (2013) Ambulatory blood pressure monitoring: importance of sampling rate and duration - 48 versus 24 hours - on the accurate assessment of cardiovascular risk. Chronobiol Int 30:55-67

34. Crespo C, Fernández JR, Aboy M, Mojón A (2013) Clinical application of a novel automatic algorithm for actigraphy-based activity and rest period identification to accurately determine awake and asleep ambulatory blood pressure parameters and cardiovascular risk. Chronobiol Int 30:43-54

35. Bakris GL (2007) Pharmacological augmentation of endotheliumderived nitric oxide synthesis. J Manag Care Pharm 13:S9-S12
36. Wang Y, Zhang MS, Liu Y (2013) Nebivolol treatment improves resistant arterial function and reduces ventricular hypertrophy and angiotensin II in spontaneously hypertension rats. J ReninAngiotensin-Aldosterone Syst 14:146-155

37. Zhao P, Xu P, Wan C, Wang Z (2011) Evening versus morning dosing regimen drug therapy for hypertension. Cochrane Database Syst Rev, Issue 10, Art. no.: CD004184. DOI: 10.1002/ 14651858.CD004184.pub2

38. Ayala DE, Moyá A, Crespo JJ et al (2013) Circadian pattern of ambulatory blood pressure in hypertensive patients with and without type 2 diabetes. Chronobiol Int 30:99-115

39. Moyá A, Crespo JJ, Ayala DE et al (2013) Effects of time-of-day of hypertension treatment on ambulatory blood pressure and clinical characteristics of patients with type 2 diabetes. Chronobiol Int 30: 116-131 